\title{
Chrono-Nutrition for Pregnant Women: Metabolic and Pediatric Insights
}

\author{
Akbar Nikkhah ${ }^{1,2 *}$ \\ ${ }^{1}$ Ferdows Pars Agri-Livestock Holding Co., Tehran, Iran \\ ${ }^{2}$ Faculty of Veterinary Medicine, University of Tehran, Iran \\ *Corresponding author: Akbar Nikkhah, Faculty of Veterinary Medicine, University of Tehran, Iran
}

\begin{abstract}
Pregnant women undergo metabolic adaptations and challenges that predispose them to a variety of complications. The objective of this article was to highlight the importance of eating timing and especially limited evening eating on optimizing pregnancy status and pediatric health and welfare. Because of limited evening and nocturnal metabolic capacity to handle nutrients (e.g., glucose), pregnant women are recommended to take smaller and earlier evening meals and instead distribute their main food meals over the course of morning and day hours. Such a food intake regimen would considerably reduce diabetes and obesity risks and improve upcoming lactation performance and pediatric health and welfare.
\end{abstract}

Keywords: Eating Timing; Pregnant Woman; Chrono-Nutrition; Pediatric Health

\section{Philosophy}

Chrono-nutrition is emerging as a new research area with growing interest (1). Chrono-nutrition deals with optimizing eating timing. The goal of chrono-nutrition is to synchronize eating patterns with circadian physiological rhythms of human metabolism $(2,3)$. This synchrony aims to reduce risks from a multitude of metabolic disorders (e.g., diabetes, obesity, cancer) and improve human health and welfare. Basically, human glucose tolerance is known to decline during evening hours and overnight. This means that cells are not as much capable to assimilate and metabolize nutrients in the evening as are they in the morning (3). In other words, if large evening meals are taken, because of incomplete and inefficient nutrient utilization by different cells, nutrient overload will occur that will in turn predispose the body to a variety of metabolic complications, such as diabetes, obesity, and metabolic syndrome.

Pregnant women undergo physiological adaptations and are already prone to metabolic challenges (e.g., diabetes and obesity) $(2,3)$. The above complications are, thus, very likely to happen in pregnant women. As such, making the evening meal smaller and taking it earlier (not later overnight) would help the pregnant body better cope with the metabolic challenges. As another complementary strategy, pregnant women are recommended to take several small evening meals made of mainly fruits and vegetables instead of starch and fats. This practice would be predicted to improve insulin action and prevent obesity. Improving evening nutrient and hormonal metabolism by evening exercise has also been advised (4). The goal has been to optimize circadian timing of eating and exercise towards optimal lifestyle. Optimizing meal properties is a new research area with many unanswered questions. Future research is required to address different aspects of this promising field of study.

\section{Conclusion}

Smaller and earlier evening meals made of mainly fruits and vegetables instead of starch and fat are recommended for pregnant women to help improve intermediary metabolism and reduce metabolic complications. These effects will result in improved lactation physiology and pediatric health and welfare. 


\section{Acknowledgments}

Thanks to Ferdows Pars Agricultural and Livestock Holding Co., (Tehran, Iran) for supporting the author's programs of optimizing science education in the third millennium.

\section{References}

1. Almoosawi S, S Vingeliene, LG Karagounis, GK Pot (2016) Chrononutrition: a review of current evidence from observational studies on global trends in time-of-day of energy intake and its association with obesity. Proc Nutr Societ 75(4): 487-500.

2. Nikkhah A (2016) Preventing maternal and pediatric obesity by optimizing meal properties. Pediat Health Res 1(1): e102.

3. Nikkhah A (2015) Synchronized rhythms of exercise and eating: A novel public program to reduce maternal and pediatric diabetes. Maternal and Paediat Nutr J 1: e101.

4. Nikkhah A (2019) Circadian timing of eating and exercise towards pptimal lifestyle: A postmodern approach. Acta Sci Nutr Health 3(5): 168-169.

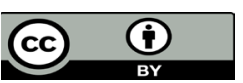

This work is licensed under Creative Commons Attribution 4.0 License

To Submit Your Article Click Here:

Submit Article

DOI: 10.32474 /PAPN.2020.03.000151

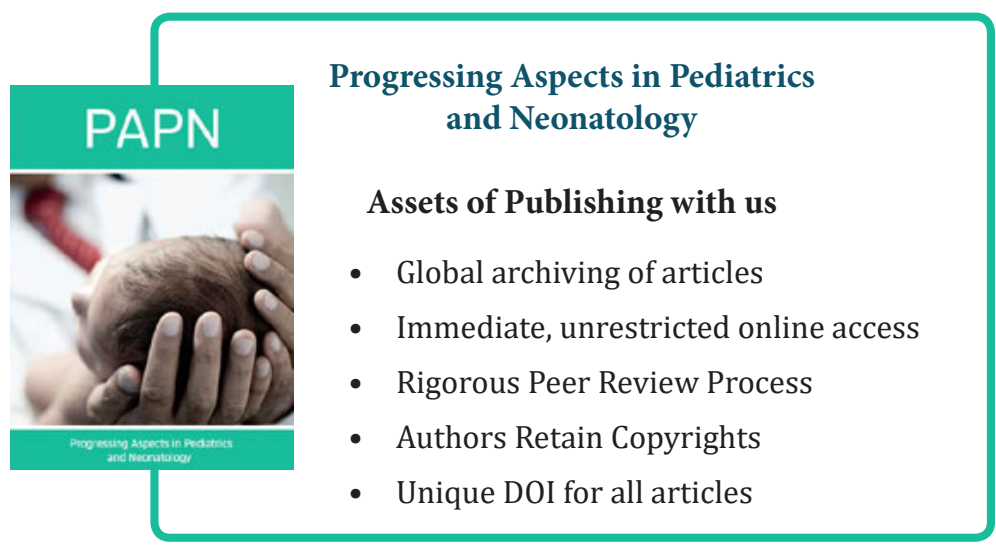

